\title{
Chemical and Organic Farming in India: An Overview
}

\author{
V. Gurumoorthy \\ Senior fellow, ICSSR, Madras Institute of Development Studies, Chennai, Tamil Nadu, India \\ E-mail: vgmindia54@gmail.com
}

\begin{abstract}
India ranks second worldwide in farm outputs. As per 2018, agriculture employed $50 \%$ of the Indian work force and contributed $17-18 \%$ to country's GDP. India exported $\$ 38$ billion worth of agricultural products in 2013, making it the seventh largest agricultural exporter worldwide and the sixth largest net exporter. Chemical fertilizers are major inputs of scientific agriculture. India is one of the major countries in the production and consumption of fertilizers. Fertilizer consumption was less than 1 million tones before the mid1960s. With the introduction of high-yielding variety (HYV) seeds, there was acceleration in the growth of fertilizer consumption. The Government of India has also implemented the National Programme for Organic Production (NPOP). The national programme involves the accreditation programme for Certification Bodies, standards for organic production, promotion of organic farming etc. As on 31st March 2018, total area under organic certification process (registered under National Programme for Organic Production) is $\mathbf{3 . 5 6}$ million Hectare (2017-18). Against this backdrop, the present study is undertaken to present an overview of chemical and organic farming in India.
\end{abstract}

Keywords: Organic Farming, Inorganic Farming, Current Status.

\section{INTRODUCTION}

1.

The history of Agriculture in India date back to Indus Valley Civilization and even before that in some places of southern India. India ranks second worldwide in farm outputs. As per 2018, agriculture employed $50 \%$ of the Indian work force and contributed $17-18 \%$ to country's GDP. India exported \$38 billion worth of agricultural products in 2013, making it the seventh largest agricultural exporter worldwide and the sixth largest net exporter. Being an organic country originally, the trend changed when the production did not meet the growing demand and the onset of food scarcity. A shift to chemical farming started in the form of Green revolution in the 1960's.Organic fertilizers were replaced by chemical fertilizers and pesticides. However, the Green Revolution, within a few years showed its impact. The land started losing its fertility, pests became immune to fertilizers, health hazards were increased.

As a result the need for organic farming has been realized and with the Government initiatives, it is being practiced again in India.

Against this backdrop, the present study is undertaken to present an overview of chemical and organic farming in India.

\section{OBJECTIVES OF THE STUDY}

1. To present an overview of chemical and organic farming in India.

2. To summarize and conclude the facts of organic and inorganic farming.

\section{STATUS OF CHEMICAL FARMING IN INDIA}

Chemical fertilizers are major inputs of scientific agriculture. India is one of the major countries in the production and consumption of fertilizers.

Fertilizer consumption was less than 1 million tones before the mid-1960s. With the introduction of high-yielding variety (HYV) seeds, there was acceleration in the growth of fertilizer consumption.

TABLE I STATE WISE DEMAND OF CHEMICAL PESTICIDESDURING 2014-15 TO 2018-19

\begin{tabular}{|c|l|c|c|c|c|c|}
\hline Sl. No. & \multicolumn{1}{|c|}{ States/UTs } & $\mathbf{2 0 1 4 - 1 5}$ & $\mathbf{2 0 1 5 - 1 6}$ & $\mathbf{2 0 1 6 - 1 7}$ & $\mathbf{2 0 1 7 - 1 8}$ & 2018-19 \\
\hline 1 & Andhra Pradesh & 4350 & 4350 & 4150 & 4000 & 3950 \\
\hline 2 & Bihar & 1003 & 1011 & 1147 & 1147 & 1147 \\
\hline 3 & Chhattisgarh & $N R$ & $N R$ & $N R$ & $N R$ & 5642 \\
\hline 4 & Goa & 13 & 43 & $N R$ & 29 & $N R$ \\
\hline 5 & Gujarat & 6260 & 2300 & 1920 & 1907 & 2103 \\
\hline 6 & Haryana & 4200 & 4200 & 4200 & 4200 & 4200 \\
\hline 7 & Himachal Pradesh & 440 & 462 & 787 & 638 & 628 \\
\hline
\end{tabular}




\begin{tabular}{|c|c|c|c|c|c|c|}
\hline 8 & Jharkhand & 683 & 690 & 690 & 650 & 625 \\
\hline 9 & Karnataka & 1800 & 1900 & 1900 & 1900 & 1900 \\
\hline 10 & Kerala & 1149 & 1149 & 1011 & 805 & 961 \\
\hline 11 & Madhya Pradesh & 825 & 811 & 809 & 706 & 650 \\
\hline 12 & Maharashtra & 9726 & 16327 & 16327 & 15004 & 15704 \\
\hline 13 & Orissa & 1084 & 990 & 990 & 990 & 1189 \\
\hline 14 & Punjab & 6370 & 6376 & 6374 & 6374 & 5765 \\
\hline 15 & Rajasthan & 2525 & 2350 & 2325 & 2325 & 2330 \\
\hline 16 & Tamil Nadu & 2141 & 2141 & 2160 & 2160 & 2122 \\
\hline 17 & Telangana & 4320 & 4135 & $N R$ & 5550 & 5642 \\
\hline 18 & Uttar Pradesh & 10453 & 10854 & 10667 & 10676 & 11031 \\
\hline 19 & Uttarakhand & 264 & 277 & 285 & 280 & 256 \\
\hline 20 & West Bengal & 3800 & 4276 & 4080 & 4125 & 4125 \\
\hline \multicolumn{2}{|c|}{ SUB TOTAL } & 63420 & 66606 & 62004 & 65757 & 72515 \\
\hline \multicolumn{7}{|c|}{ North-Eastern } \\
\hline 21 & Arunachal Pradesh & 18 & 17 & 18 & $N R$ & 5 \\
\hline 22 & Assam & 215 & $N R$ & $N R$ & 241 & 347 \\
\hline 23 & Manipur & 35 & 34 & $N R$ & 28 & $N R$ \\
\hline 24 & Meghalaya & 30 & $N R$ & $N R$ & $N R$ & $N R$ \\
\hline 25 & Mizoram & 1162 & $N R$ & 1215 & $N R$ & 171 \\
\hline 26 & Nagaland & 25 & 26 & 26 & 26 & 28 \\
\hline 27 & Sikkim & $N R$ & $N R$ & $N R$ & $N R$ & $N R$ \\
\hline 28 & Tripura & 510 & 393 & 428 & 370 & $N R$ \\
\hline \multicolumn{2}{|r|}{ SUB TOTAL } & 1995 & 470 & 1687 & 665 & 550 \\
\hline \multicolumn{7}{|c|}{ Union Territories } \\
\hline 29 & A \& N Islands & 4 & $N R$ & $N R$ & $N R$ & $N R$ \\
\hline 30 & Chandigarh & $N R$ & $N R$ & $N R$ & $N R$ & $N R$ \\
\hline 31 & $\begin{array}{l}\text { Dadra \& Nagar } \\
\text { Haveli }\end{array}$ & $N R$ & $N R$ & $N R$ & $N R$ & $N R$ \\
\hline 32 & Daman \& Diu & $N R$ & $N R$ & $N R$ & $N R$ & $N R$ \\
\hline 33 & Delhi & $N R$ & $N R$ & $N R$ & $N R$ & 130 \\
\hline 34 & Jammu \& Kashmir & 2015 & 1964 & 2181 & 2290 & 2545 \\
\hline 35 & Ladakh & $N R$ & $N R$ & $N R$ & $N R$ & $N R$ \\
\hline 36 & Lakshadweep & $N R$ & $N R$ & $N R$ & $N R$ & $N R$ \\
\hline 37 & Pondicherry & 48 & 50 & 50 & 50 & 48 \\
\hline \multicolumn{2}{|r|}{ SUB TOTAL } & 53 & 50 & 50 & 50 & 178 \\
\hline \multicolumn{2}{|c|}{ GRAND TOTAL } & 65468 & 67126 & 63740 & 66472 & 73244 \\
\hline
\end{tabular}

The above table shows the state wise and Union Territory wise demand of fertilizers in India. It could be seen that certain states like Maharashtra have increased demand for fertilizers over the years. States like Madhya Pradesh have recorded decrease in the demand of chemical fertilizers in the selected years. Thus, the table exhibits the overall demand for the fertilizers in all the states and union territories of India. 
TABLE II CONSUMPTION OF CHEMICAL PESTICIDES IN VARIOUS STATES/UTS DURING 2014-15 TO 2018-19

\begin{tabular}{|c|c|c|c|c|c|c|}
\hline Sl. No. & States/UTs & 2014-15 & 2015-16 & 2016-17 & 2017-18 & 2018-19 \\
\hline 1 & Andhra Pradesh & 4050 & 2713 & 2015 & 1738 & 1689 \\
\hline 2 & Bihar & 787 & 831 & 790 & 840 & 850 \\
\hline 3 & Chhattisgarh & 1589 & 1625 & 1660 & 1685 & 1770 \\
\hline 4 & Goa & 12 & 48 & 22 & 24 & 25 \\
\hline 5 & Gujarat & 1730 & 1980 & 1713 & 1692 & 1608 \\
\hline 6 & Haryana & 4070 & 4100 & 4050 & 4025 & 4015 \\
\hline 7 & Himachal Pradesh & 379 & 450 & 341 & 467 & 322 \\
\hline 8 & Jharkhand & 650 & 493 & 541 & 619 & 646 \\
\hline 9 & Karnataka & 1793 & 1434 & 1288 & 1502 & 1524 \\
\hline 10 & Kerala & 910 & 1123 & 895 & 1067 & 995 \\
\hline 11 & Madhya Pradesh & 696 & 732 & 694 & 502 & 540 \\
\hline 12 & Maharashtra & 8663 & 11665 & 13496 & 15568 & 11746 \\
\hline 13 & Orissa* & 1278 & 994 & 1050 & 1633 & 1609 \\
\hline 14 & Punjab & 5689 & 5743 & 5843 & 5835 & 5543 \\
\hline 15 & Rajasthan & 2694 & 2475 & 2269 & 2307 & 2290 \\
\hline 16 & Tamil Nadu & 2096 & 2096 & 2092 & 1929 & 1901 \\
\hline 17 & Telangana & 2806 & 993 & 3436 & 4866 & 4894 \\
\hline 18 & Uttar Pradesh & 9736 & 10457 & 10614 & 10824 & 11049 \\
\hline 19 & Uttarakhand & 172 & 217 & 198 & 210 & 195 \\
\hline 20 & West Bengal & 3060 & 3712 & 2624 & 2982 & 3190 \\
\hline & Sub Total & 52859 & 53881 & 55631 & 60316 & 56402 \\
\hline \multicolumn{7}{|c|}{ North-Eastern } \\
\hline 21 & Arunachal Pradesh & 18 & 17 & 18 & $N R$ & 5 \\
\hline 22 & Assam & 190 & 185 & 306 & 241 & 256 \\
\hline 23 & Manipur & 31 & 30 & 33 & 27 & NR \\
\hline 24 & Meghalaya & 28 & $N R$ & $N R$ & $N R$ & NR \\
\hline 25 & Mizoram & 805 & $N R$ & 9 & $N R$ & 26 \\
\hline 26 & Nagaland & 20 & 20 & 20 & 20 & 21 \\
\hline 27 & Sikkim & Organic State & Organic State & Organic State & Organic State & Organic State \\
\hline 28 & Tripura & 346 & 293 & 298 & 330 & 349 \\
\hline & Sub Total & 1437 & 544 & 684 & 617 & 657 \\
\hline \multicolumn{7}{|c|}{ Union Territories } \\
\hline 29 & Andaman \& Nicobar & 8 & $N R$ & $N R$ & $N R$ & NR \\
\hline 30 & Chandigarh & $N R$ & $N R$ & $N R$ & $N R$ & NR \\
\hline 31 & $\begin{array}{l}\text { Dadra \& Nagar } \\
\text { Haveli }\end{array}$ & $N R$ & $N R$ & $N R$ & $N R$ & NR \\
\hline 32 & Daman \& Diu & $N R$ & $N R$ & $N R$ & $N R$ & NR \\
\hline 33 & Delhi & $N R$ & $N R$ & 88 & $N R$ & 110 \\
\hline 34 & Jammu \& Kashmir & 1921 & 2251 & 2188 & 2430 & 2459 \\
\hline 35 & Ladakh & - & - & - & - & Formed in Oct,19 \\
\hline 36 & Lakshadweep & - & $N R$ & $N R$ & $N R$ & NR \\
\hline \multirow[t]{3}{*}{37} & Pondicherry & 42 & 43 & 43 & 43 & 42 \\
\hline & Sub Total & 1971 & 2295 & 2319 & 2473 & 2611 \\
\hline & Grand Total & 56268 & 56720 & 58634 & 63406 & 59670 \\
\hline
\end{tabular}


The consumption of pesticides in various states and Union Territories from 2014-15 to 2018-19 has been presented in the above table. Compared to the demand of chemical fertilizers, the consumption of fertilizers shows a decreasing trend in major states.

Due to the growing awareness of organic farming and soil deterioration the consumption of chemical fertilizers in various states of India presents a different scenario.

\section{STATUS OF ORGANIC FARMING IN INDIA}

As per the available statistics, India's rank in terms of World's Organic Agricultural land was 9th and in terms of total number of producers was 1st as per 2018 data (Source: FIBL \& IFOAM Year Book 2018).The Government of India has implemented the National Programme for Organic Production (NPOP). The national programme involves the accreditation programme for Certification Bodies, Standards for organic production, promotion of organic farming etc. As on 31st March 2018, total area under organic certification process (registered under National Programme for Organic Production) is 3.56 million Hectare (2017-18). This includes 1.78 million ha (50\%) cultivable area and another 1.78 million Hectare (50\%) for wild harvest collection.

Among all the states, Madhya Pradesh has covered largest area under organic certification followed by Rajasthan, Maharashtra and Uttar Pradesh.

During 2016, Sikkim has achieved a remarkable distinction of converting its entire cultivable land (more than 76000 ha) under organic certification.

TABLE III INSTALLED CAPACITY, PRODUCTION AND DISPATCHES OF BIO-FERTILIZER IN INDIA

\begin{tabular}{|c|c|c|c|c|}
\hline Year & $\begin{array}{c}\text { Installed production } \\
\text { Capacity (tonnes) }\end{array}$ & $\begin{array}{c}\text { Total Production } \\
\text { (tonnes) }\end{array}$ & $\begin{array}{c}\text { Total Dispatches } \\
\text { (tonnes) }\end{array}$ & $\begin{array}{c}\text { Unutilized capacity } \\
\text { percentage }\end{array}$ \\
\hline $1992-93$ & 5400.5 & 2005 & 1600.01 & 62.87 \\
\hline $1993-94$ & 6125.5 & 3084 & 2914 & 49.65 \\
\hline $1994-95$ & 8114.5 & 5800.5 & 4988.9 & 28.52 \\
\hline $1995-96$ & 10680.4 & 6692.3 & 6288.3 & 37.34 \\
\hline $1996-97$ & 12647 & 7406.6 & 6681.4 & 41.44 \\
\hline $1998-99$ & 16446 & 5972.1 & 5065.5 & 63.69 \\
\hline $2001-02$ & 15439.0 & 9019.2 & 8429.3 & 41.59 \\
\hline $2002-03$ & 18679.5 & 7181.7 & 7029.9 & 61.55 \\
\hline $2003-04$ & 18632 & 8701.4 & 8357 & 53.30 \\
\hline $2004-05$ & NA & 10479 & 10428 & - \\
\hline $2005-06$ & NA & 11752 & 11358 & - \\
\hline $2006-07$ & 43495 & 15871 & 15745 & 63.51 \\
\hline $2007-08$ & 67162 & 20111 & 20100 & 70.06 \\
\hline $2008-09$ & 68804 & 25065 & 25000 & 63.57 \\
\hline $2009-10$ & 68078 & 20040.3 & 20000 & 70.56 \\
\hline
\end{tabular}

The Installed Capacity, Production and Dispatches of Bio-Fertilizer during various years have been compiled and presented in the above table.1992-2010 bio fertilizer production capacity has been increased tremendously along with total production and total utilization. The concern towards sustainable development has made organic farming a viable alternative for chemical farming. The increase in the production of bio fertilizer stands testimony to the fact that the organic farming has been booming over the years.

\section{CONCLUSION}

The study has presented the current status of both organic and inorganic inputs in a brief manner. In India, in last three decades, food grain productions increased from 50 million tons to 170 million tons. This was with increased intensive farming with modern agricultural technologies ushered in Green revolution. However, due to its aggressive usage, now organic farming is preferred. It is worthwhile to note that a balance has been created between both the inputs by the Government initiatives. With the right amount of both the inputs, India is all set to lead as the agricultural giant in terms of production, consumption and export across the globe.

\section{REFERENCES}

[1] Kumara Charyulu Deevi and Subho Biswas. 2011. Organic Input Production and Marketing in India, Allied Publisher Pvt Ltd, 13-15.

[2] Retrieved from www.en.m.wikipedia.org.

[3] Retrieved from www.wwoofindia.org

[4] Retreived from www.informaticcsjournals.com. 